\title{
GAIA Level 3 Intrapartum Stillbirth
}

National Cancer Institute

\section{Source}

National Cancer Institute. GAIA Level 3 Intrapartum Stillbirth. NCI Thesaurus. Code C128024.

GAIA Level 3 Intrapartum Stillbirth is defined by four criteria: first, delivery of an infant who is reported to have no of signs of life at birth, including the following: no spontaneous movements, no umbilical cord pulse, no heartbeat, no crying, no spontaneous respirations or chest movement, and full body cyanosis; second, evidence of a live fetus prior to the onset of labor (maternal report of fetal movement prior to the onset of labor OR auscultation of fetal heart tones). NOTE: in the absence of evidence of a live fetus prior to the onset of labor, the fetal death should be reported as a stillbirth or an antepartum stillbirth; third, a non-attended delivery followed by a post-delivery physical examination that is performed by a health care professional who meets the standard of care in the immediate health care setting, and that is consistent with intrapartum death, or a verbal history that is provided by a trained health care provider, a non-medical witness, or the mother of the fetus that documents a fetus born with no signs of life and who was unresponsive to resuscitation efforts immediately after birth; fourth, gestational age within the pre-defined range for selected stillbirth definition as assessed by maternal and/or fetal parameters (level 2-3 in GA assessment algorithm). 